Projets

de paysage

\section{Projets de paysage}

Revue scientifique sur la conception et l'aménagement de l'espace

9 | 2013

Le paysage a t-il imposé sa loi ?

\title{
Les photographies du paysage : quelles analyses des dynamiques paysagères?
}

Landscape Photographs: What Analyses for Landscape Dynamics?

\section{Caroline Guittet et Laurence Le Dû-Blayo}

\section{OpenEdition}

\section{Journals}

Édition électronique

URL : http://journals.openedition.org/paysage/12477

DOI : $10.4000 /$ paysage. 12477

ISSN : 1969-6124

\section{Éditeur :}

École nationale supérieure du paysage de Versailles-Marseille, Institut national des sciences appliquées Centre Val de Loire - École de la nature et du paysage, École nationale supérieure d'architecture et de paysage de Bordeaux, École nationale supérieure d'architecture et de paysage de Lille, Agrocampus Angers

\section{Référence électronique}

Caroline Guittet et Laurence Le Dû-Blayo, « Les photographies du paysage : quelles analyses des dynamiques paysagères? », Projets de paysage [En ligne], 9 | 2013, mis en ligne le 20 décembre 2013, consulté le 18 novembre 2020. URL : http://journals.openedition.org/paysage/12477 ; DOI : https:// doi.org/10.4000/paysage. 12477

Ce document a été généré automatiquement le 18 novembre 2020.

Projets de paysage 


\title{
Les photographies du paysage : quelles analyses des dynamiques paysagères?
}

\author{
Landscape Photographs: What Analyses for Landscape Dynamics?
}

Caroline Guittet et Laurence Le Dû-Blayo

1 De tout temps, les paysages ont connu des mutations qui ont pu être profondes à certains moments. Liés aux facteurs naturels et/ou humains, ces changements interviennent sur l'espace physique et sur les représentations sociales des populations de manière concomitante ou isolée, de manière visible ou invisible à des échelles spatiotemporelles variables. L'analyse des dynamiques paysagères permet d'appréhender ces mécanismes d'évolution.

2 Reconnus juridiquement (loi Paysage, 1993, Convention européenne du paysage, 2000), les paysages ordinaires, devenus « bien commun » (Sgard, 2010), doivent être identifiés et caractérisés (avec les atlas du paysage notamment) par les collectivités territoriales. Cette qualification des paysages s'opère également par l'analyse des dynamiques paysagères passées pour mieux appréhender et orienter leurs évolutions futures au sein des politiques territoriales. Répondant à cela, l'observatoire photographique national du paysage (OPNP), lancé en 1991 par le ministère de l'Environnement, a pour objectif « de constituer un fonds de séries photographiques qui permette d'analyser les mécanismes et les facteurs de transformations des espaces ainsi que les rôles des différents acteurs qui en sont la cause de façon à orienter favorablement l'évolution du paysage" (Meeddm ${ }^{1}, 2009$, p. 8). Ce fonds photographique peut bénéficier d'une approche rétrospective et/ou prospective. Pour la première démarche, il s'agit de rephotographier aujourd'hui, selon des méthodes rigoureuses, des points de vue issus de photographies anciennes pour reconstituer des évolutions paysagères. Quant à l'approche prospective, elle a pour finalité de suivre des évolutions à venir. La méthode mise en place est la suivante : un comité de pilotage constitué d'élus, de professionnels et d'associations détermine les préoccupations paysagères actuelles et à venir sur le territoire. Après une première campagne photographique menée par le photographe 
professionnel, le comité de pilotage se concerte afin de sélectionner une quarantaine de points de vue initiaux représentatifs des problématiques paysagères déterminées en amont. À partir de modalités de reconduction définies (Meeddat, 2008), ces points de vue, formant un itinéraire, sont reconduits à des intervalles de temps réguliers fixés en fonction des typologies des paysages suivis. La démarche n'est pas nouvelle (Grison, 1998), déjà expérimentée par les services de restauration des Terrains en montagne à partir de 1882, puis les services forestiers en Italie, en Suisse et en Australie, elle se développe massivement dans les années 1960 aux États-Unis (Carré, Métailié, 2008). La photographie de paysage a dès son apparition suscité l'intérêt des différents regards (artistes, naturalistes, géographes, etc.) ; plus encore aujourd'hui avec l'avènement du numérique et « avec la montée en puissance des préoccupations paysagères » (Dérioz et al., 2010, p. 2). Ainsi depuis une dizaine d'années, en parallèle des 19 itinéraires de l'OPNP, de nouveaux Observatoires photographiques du paysage (OPP) locaux émergent en France (Meeddm, 2009). La région Bretagne illustre cette diversité. On y trouve deux itinéraires de l'OPNP (OPP du CAUE ${ }^{2}$ des Côtes d'Armor, OPP du parc naturel régional d'Armorique) et deux OPP locaux (OPP du Siagm ${ }^{3}$ et OPP du Pays de Saint-Brieuc).

Figure 1. La répartition des OPP en Bretagne

Carte des Observatoires photographiques du paysage en Bretagne

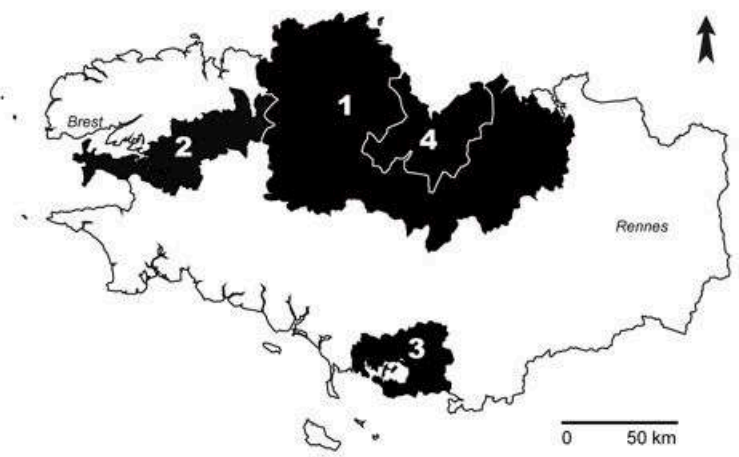

Les OPP labellisés

1 OPP du Conseil d'Architecture, d'Urbanisme et de l'Environnement

des Côtes d'Armor (Date de création: 1994)

2 OPP du Parc Naturel Régional d'Armorique (Date de création:1997)

Les OPP locaux

3 OPP du Syndicat Intercommunal d'Aménagement du Golfe du Morbihan

(Date de création: 2004)

4 OPP du Syndicat Mixte du Pays de Saint-Brieuc (Date de création: 2011)

Cette multiplication des OPP est à l'origine de nombreuses séries photographiques, qui restent souvent sous-exploitées faute d'accessibilité et faute de méthodes d'analyses stabilisées. La région Bretagne, engagée dans la mise en place de politiques paysagères (Le Dû-Blayo, 2007), s'investit dans la conception d'outils par le biais d'une convention avec le laboratoire Eso-Rennes afin de sensibiliser les acteurs locaux aux dynamiques paysagères et à la valorisation des OPP. Née de cette volonté, la commande publique présentée ici se traduit par une animation virtuelle sur l'évolution des dynamiques paysagères en Bretagne à travers des blocs diagrammes ${ }^{4}$. Un des trois blocs diagrammes rend compte de l'évolution des dynamiques paysagères urbaines en Bretagne. Il se déploie dans le temps via cinq phases chronologiques allant de 1900 à 2012. Chaque phase s'appuie sur un corpus iconographique construit à partir des OPP et de 
recherches complémentaires dans les archives départementales, les collections privées et les fonds des institutions partenaires.

4 Nous nous attacherons dans cette contribution à expliciter les apports et les limites des corpus photographiques dans la connaissance des dynamiques paysagères à travers des approches multiscalaires; ce qui nous conduira à interroger les conditions de production et d'utilisation des OPP.

5 Nous présenterons tout d'abord différentes expérimentations méthodologiques qui ouvrent des perspectives pour l'analyse des dynamiques urbaines à partir des séries photographiques. Puis, après l'examen critique du corpus iconographique utilisé dans le cadre de l'animation, nous proposerons des pistes d'exploitation des OPP pour l'analyse des dynamiques paysagères urbaines à l'échelle régionale.

\section{État de l'art des analyses des dynamiques paysagères à partir des corpus photographiques du paysage}

6 Les potentialités des corpus photographiques en matière d'analyse des dynamiques paysagères ont déjà fait l'objet de nombreuses expérimentations dans des contextes disciplinaires variés. Les méthodes proposées ici amorcent l'analyse du corpus photographique par l'identification des changements affectés aux éléments de paysage ${ }^{5}$.

\section{L'approche qualitative diachronique}

7 L'approche qualitative diachronique est la plus communément utilisée pour analyser les dynamiques paysagères à travers les séries photographiques. "La démarche suivie repose sur l'analyse multiscalaire et la méthode régressive » partant du présent pour reconstituer les paysages passés (Métailié, 1997, p. 91). La première étape consiste à choisir les éléments de paysage et à décrire les structures paysagères ${ }^{6}$ à partir d'une lecture de terrain. Ces éléments et structures sont ensuite "rétro-observés " par une description fine des séries diachroniques (Michelin, 1995; Carré et Métailié, 2008; Davasse et al., 2012 ; Henry, 2012). L'interprétation photographique est ensuite croisée avec d'autres données afin de généraliser les hypothèses. L'enquête auprès des acteurs locaux, avec comme support les photographies répétées, vise «à cerner la sensibilité des habitants aux transformations paysagères" (Davasse et al., op.cit., p. 13). Ces recherches explorent donc une écriture du passé paysager par la connexion entre documents et témoignages. Méthode rigoureuse et archivistique, elle met en scène un « récit interprétatif » (Henry, op. cit., p. 138) où la sélection des documents s'effectue selon leur disponibilité avec des hiatus thématiques, chronologiques et géographiques.

\section{La quantification des dynamiques paysagères sur les séries photographiques}

Un certain nombre d'études ont une approche quantitative des dynamiques paysagères à partir des séries photographiques. Deux exemples peuvent être ici présentés. Le contrat rivière Semois-Semoy, dans le cadre de l'OPP, teste une grille d'éléments à observer à partir de grandes thématiques (hydrologie, agriculture, etc.). Les 
changements d'éléments de paysage (apparition-disparition) sont répertoriés par l'expert puis synthétisés dans cette grille (Lobet et al., 2006). À partir de cette base, des tendances d'évolutions sont décrites. Cette grille présente l'intérêt d'inventorier précisément les éléments et de décrire des dynamiques complexes. Cependant, la quantification des éléments décontextualisés de l'ensemble photographique permet difficilement d'établir les interrelations entre les éléments et les dynamiques.

Dans le cadre de son stage au Bureau des paysages, Adrien Coutanceau, par le jeu des " 7 erreurs ", appose un aplat de couleur sur chaque élément qui a évolué entre chaque prise de vue. (Coutanceau, 2011). Une image est ensuite conçue synthétisant les changements identifiés sur l'ensemble de la série. À partir de celle-ci, une nouvelle image modélise les dynamiques. Dans les deux cas, ces méthodes permettent d'extraire les changements analysés sur les séries photographiques et apportent des indices sur des dynamiques paysagères émergentes.

\section{La quantification de la surface des éléments paysagers sur les séries photographiques}

D'autres travaux s'orientent vers une approche plus systématique pour quantifier l'évolution de la surface occupée par les éléments du paysage photographiés comme développés à Unité mixte de recherche "Théoriser et modéliser pour aménager " (UMR ThéMA)․ Étudiant le Spitzberg occidental, Madeleine Griselin et Serge Ormaux installent un dispositif photographique complexe ${ }^{8}$ sur l'ensemble de la zone du bassin Lovén est avec des prises de vue systématiques (Griselin, Ormaux, 2005). Ce corpus conséquent est ensuite traité par un outil informatique pour mesurer notamment l'évolution des surfaces enneigées ou englacées. Ces espaces, par leur « caractère épuré et minéral » (ibid., p. 3) facilitent l'analyse automatisée.

11 Les paysages anthropisés complexes tels que ceux photographiés dans les OPP présentent une variété d'éléments et de superpositions qui ne peuvent pas être extraits de manière automatique comme l'exposent les travaux sous la direction d'Yves Luginbühl (1999). Chaque photographie ici est décomposée par plan puis pour chaque plan, on mesure la surface couverte de chaque élément de paysage retranscrite en pourcentage de la surface totale. Les fiches issues de ce travail sont transposées dans un logiciel pour « analyser les divers types de transformations, les plus marquants, les plus durables dans les séries photographiques analysées » (ibid., p. 11). Le logiciel prend en considération l'intégralité des changements comme ceux du cycle saisonnier.

Les travaux de Pierre Enjelvin et de Christian Guy ${ }^{9}$ couplent une analyse par objet et une analyse par plan. L'analyse par objet consiste à décrire les changements paysagers affectant les objets définis en amont. L'analyse par plan réside dans la décomposition de la photographie par plans, eux-mêmes décomposés par les différentes matières de paysage (eau, sol, etc.). Pour chaque photographie, «les matières de paysages sont quantifiées en pourcentage d'occupation de la surface photographique dans chaque plan $^{10} »$. Ce croisement des deux méthodes participe à la facilitation des analyses photographiques par une tierce personne.

13 Les traitements automatisés d'images ont l'avantage d'exploiter un important fonds iconographique. Néanmoins ces logiciels ne sont pas aptes à détecter certains phénomènes infimes et ils peuvent indiquer des changements majeurs en termes de surface, mais peu signifiants dans l'analyse des dynamiques paysagères. 


\section{La quantification de la surface des éléments paysagers reportée sur une carte}

14 Les travaux d'Alain Dervieux sur l'évolution des boisements en Camargue et en Languedoc ${ }^{11}$ proposent à partir de photographies diachroniques une cartographie comparative mesurant l'évolution de l'occupation du sol par les boisements du début $\mathrm{du} \mathrm{xx}^{\mathrm{e}}$ siècle à aujourd'hui. À partir d'une étude visuelle par l'identification des éléments sur la photographie, un croquis met en évidence les différents recouvrements du sol (graviers, cultures, etc.) qui seront ensuite reportés sur une carte d'état-major. Centrée sur une dynamique précise, l'analyse ignore les éléments en dehors du recouvrement du sol et les interactions possibles entre ces éléments.

Trois PNR associés ${ }^{12}$ travaillent dans ces mêmes perspectives avec une mise en cartographie des changements de l'occupation du sol analysés au préalable sur les séries de l'OPP et via des études d'occupation du sol. Cette phase est alimentée par une étude qualitative des changements affectant les éléments de paysage. La jonction de ces deux méthodes marque le passage de la vue horizontale à la vue verticale. Chaque échelle de représentation informe différemment les dynamiques paysagères avec un basculement spatial de l'élément à la structure du paysage.

Cet état de l'art expose la complémentarité entre une analyse qualitative et une analyse quantitative. L'analyse quantitative rend possible l'élaboration d'indicateurs de mesure des changements paysagers et l'analyse qualitative met en lumière des indicateurs plus complexes notamment sur les usages. Ces exemples méthodologiques montrent également que la photographie du paysage, image d'un fragment de territoire, retient seulement une partie des formes visibles et occulte complètement l'ensemble des phénomènes non perceptibles. Le contexte hors champ de la photographie, les contextes historique, politique, social sont suggérés par quelques indices de la photographie. Des documents connexes (statistiques, plans locaux d'urbanisme, etc.) et le travail de terrain (enquêtes et/ou observations) sont nécessaires pour compléter les données iconographiques. La cohérence de ces analyses dépend aussi de la qualité et des caractéristiques du corpus iconographique à disposition.

\section{Les caractéristiques du corpus photographique}

Les 240 photographies étudiées ici ont été sélectionnées dans le cadre de la commande publique pour renseigner des dynamiques paysagères urbaines en Bretagne. Ce corpus est relativement représentatif des potentiels et des limites des photographies sur les paysages.

\section{Cadrage/point de vue}

Le format de la photographie est une donnée essentielle tant dans la contrainte des informations que dans la perception de celles-ci (Sevenant, Antrop, 2011). Empruntant les typologies de "formatage » effectuées par Didier Mendibil (2008), la figure 2 classe le corpus photographique selon le cadrage des photographies ${ }^{13}$ ( $c f$. lignes de la figure 2) et selon leur point de vue ${ }^{14}$ ( $f f$. colonnes de la figure 2). La somme des deux est appelée le «format ». La variété des formats proposés est issue de l'étude de Didier Mendibil qui 
s'attache à analyser l'iconographie de la géographie française de 1839 à 1990 en décodant le langage des images. Cette méthode de classement convient à notre corpus paysager puisqu'elle traite des photographies de vues paysagères. Pour les cadrages «objectivants », les contours de l'objet photographié sont bien délimités, le cadrage mixte accentue la profondeur de champ. Tous deux explicitent l'intentionnalité du photographe dirigeant le regard (63\%). Les cadrages "subjectivants» dévoilent la sensibilité du photographe avec son environnement par les « divers effets visuels tels que le redoublement du cadre ou la liaison avec le hors-champ» (ibid., p.5). Les éléments sont moins lisibles puisqu'ils sont suggérés, coupés. $40 \%$ des séries OPP étudiées présentent des portions de rond-point, de mur, de monument.

Figure 2. Les pourcentages de $1^{\text {re }}$ occurrence représentent la part du corpus total et les pourcentages de $2^{\mathrm{e}}$ occurrence (en gras) sont la part des séries OPP étudiés

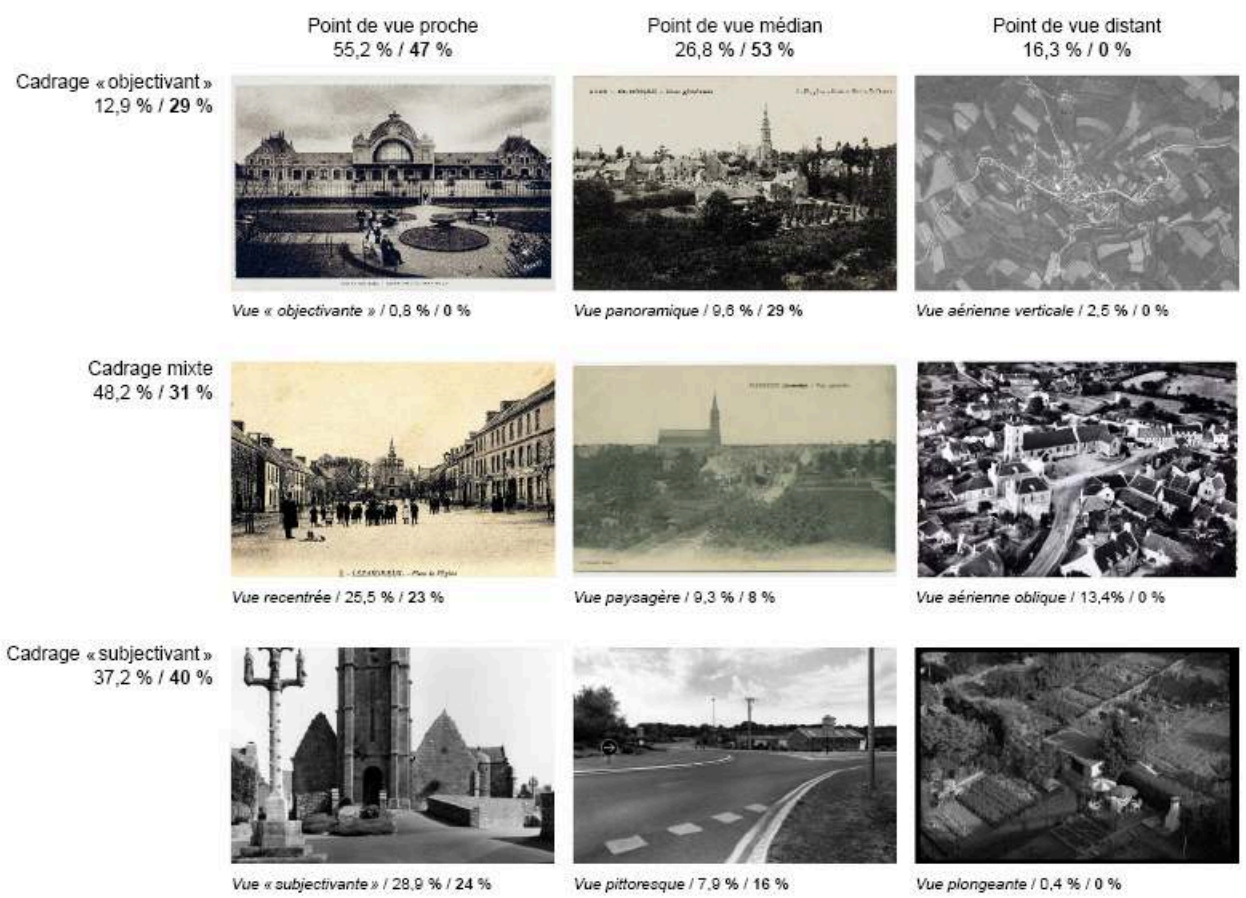

Des microespaces représentent 1,7 \% du corpus, non classés dans le tableau.

Sources (de gauche à droite) : Archives départementales des Côtes d'Armor, fonds 6 Fl, droits réservés/Archives départementales des Côtes d'Armor, fonds $6 \mathrm{FI}$, droits réservés/IGN, droits réservés/collection particulière, droits réservés/Archives départementales d'Ille-et-Vilaine, fonds $6 \mathrm{FI}$, droits réservés/Archives départementales des Côtes d'Armor, fonds $16 \mathrm{Fl}$, droits réservés/JeanChristophe Ballot, Observatoire photographique national du paysage, ministère, Itinéraire $n^{\circ} 19$, PNRA, droits réservés/Jean-Christophe Ballot, Observatoire photographique national du paysage, ministère, Itinéraire n 19, PNRA, droits réservés/CAUE 22, droits réservés.

Quant aux colonnes, elles «marquent l'effet de distance croissante entre les points de vue et les objets» (ibid., p. 5). Les points de vue proches, avec plus de $50 \%$ du corpus, donnent à voir le détail des éléments de paysage comme des édifices. Les vues recentrées et «subjectivantes » sont récurrentes dans les séries OPP facilitant l'analyse fine de l'évolution des éléments. Focalisées sur un élément, les vues "objectivantes " donnent peu d'informations pour notre étude. Les points de vue médians, avec un premier plan à quelques kilomètres, révèlent des éléments de paysage en nombre important. Ils invitent à les prendre en considération dans leur contexte (vue générale 
d'un village, entrée de ville). Les points de vue lointains « apportent un basculement de la perspective puisque d'horizontale elle devient aérienne et donc plus ou moins verticale» (ibid., p. 5). Ils permettent « de vérifier et d'affiner la corrélation entre les dynamiques paysagères perceptibles au ras du sol et les données de la vision verticale » (Carré et al., 2008, p.134.) Ces échelles de visualisation s'emboîtent entre elles, introduisant un élargissement spatial du paysage. L'analyse de ce corpus aux divers formats souligne des inégalités, des discontinuités que nous pouvons synthétiser par des hiatus temporels, spatiaux et thématiques.

\section{Les hiatus temporels}

Figure 3. Le corpus iconographique est classé selon la typologie des photographies et selon les phases chronologiques

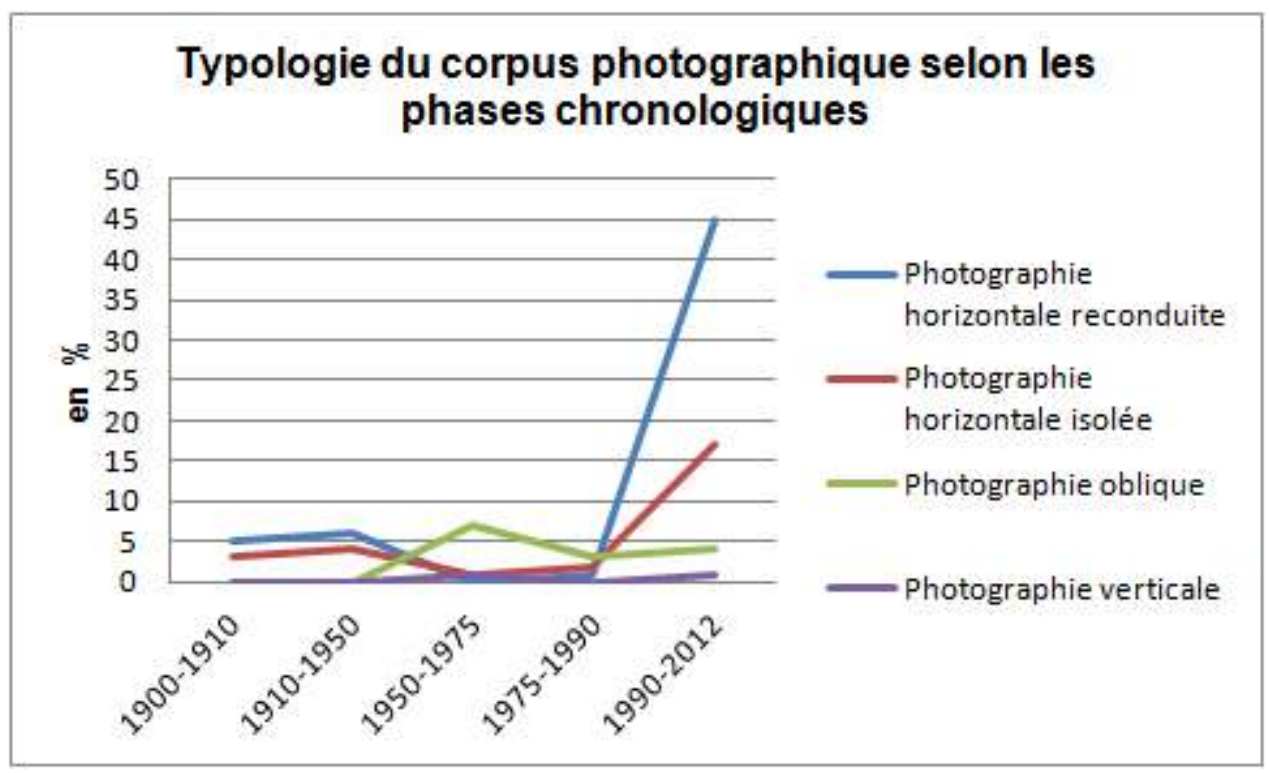

Le graphique ( $c f$. figure 2) éclaire les typologies du corpus photographique utilisé selon les périodes chronologiques. Avec des orientations rétrospectives, les couples diachroniques (29\%) illustrent les dynamiques paysagères à une échelle séculaire en majorité. Pour l'essentiel, il s'agit de cartes postales anciennes (1900-1950) issues des archives départementales ou de collections privées qui sont reconduites à l'identique dans les années 1990-2012. Le corpus est également composé de photographies aériennes obliques en plein essor à partir des années 1950 et de photographies sans reconduction issues des structures porteuses d'OPP (cf. figure 3). Ces fonds institutionnels très riches sont difficilement exploitables notamment pour les diapositives des années 1980-1990 qui sont souvent peu renseignées et non numérisées. 
Figure 4. Provenance des photographies analysées

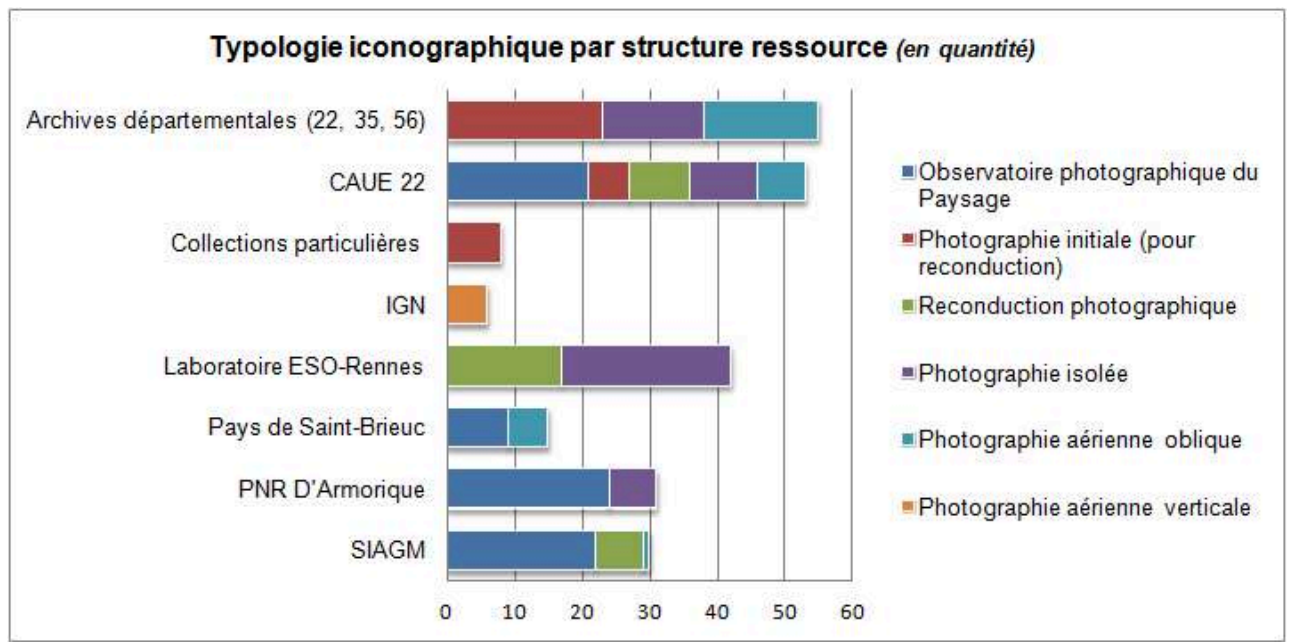

21 Néanmoins, ces photographies isolées enrichissent les séries de paysage « à un moment donné ». Le pic de la dernière période (1990-2012) annonce l'avènement des OPP. Représentant ici $32 \%$ du corpus, avec des reconductions annuelles ou pluriannuelles, ils apportent un regard contemporain sur le paysage dans des visées prospectives.

\section{Les hiatus spatiaux}

Figure 5. Répartition des photographies concentrées sur les villes moyennes et les villages de $1^{\text {re }}$ ou $2^{\text {de }}$ couronne

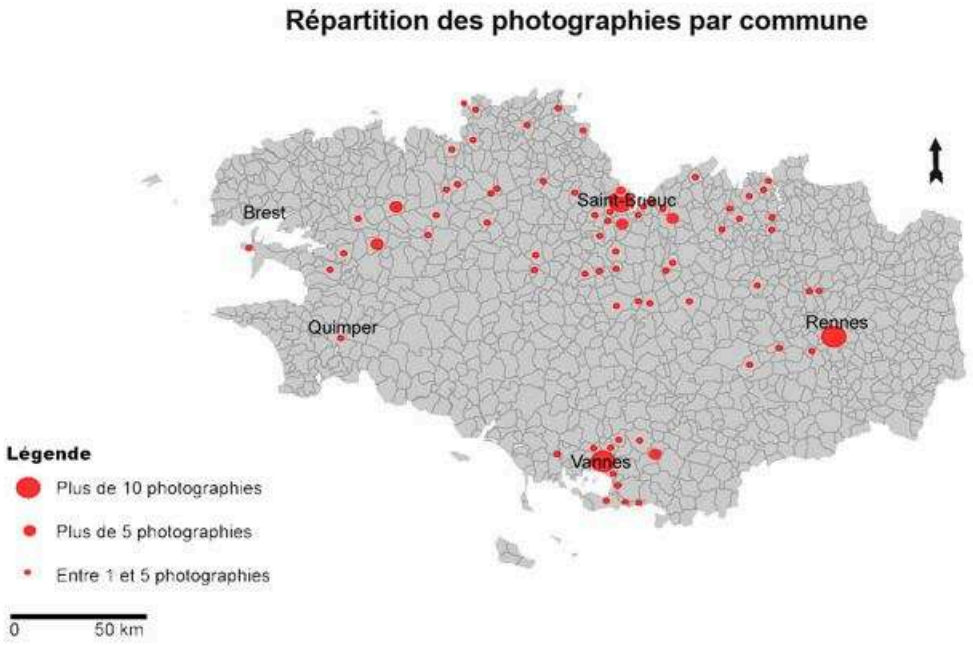

Les territoires couverts par un OPP sont bien sûr largement représentés mais avec un recouvrement discontinu. Une concentration de photographies est visible autour des villes moyennes comme Saint-Brieuc, Lamballe, Vannes et des villages de $1^{\text {re }}$ et $2^{\text {de }}$ couronnes de ces villes. La situation géographique de ces villes et villages favorise la couverture photographique diachronique et contemporaine ( $c f$. figure 4$)$. En effet, à proximité du littoral, les cartes postales illustrent ces espaces touristiques et les OPP 
veillent à ces espaces sous pression (démographie urbaine, étalement urbain, pollution, etc.). Le corpus issu des recherches sur les dynamiques paysagères de villes moyennes est représentatif de l'organisation spatiale de la Bretagne avec peu de grandes villes à l'exception de Rennes.

Les hiatus temporels soulignent l'évolution de la photographie du paysage selon le contexte social (carte postale) et les avancées technologiques (photographie aérienne). Les hiatus spatiaux marquent plus spécifiquement des partis pris dans le cadre du projet pour modéliser les dynamiques paysagères. Quoi qu'il en soit, la photographie de terrain est une donnée discrète dont la représentation spatiale est très ponctuelle, elle doit être exploitée avec précaution pour toute tentative de généralisation.

\section{Les hiatus thématiques}

Selon la période, le statut du photographe (artiste ou chargé de mission) et ses propres filtres culturels, le regard porté sur le paysage diffère. Le tableau 2 répertorie les sujets d'intérêts mis en avant dans le corpus étudié selon les périodes. Par exemple, le regard porté sur la «nature » en ville au début du $\mathrm{xx}^{\mathrm{e}}$ siècle s'apparente aux jardins hérités du XIX ${ }^{e}$ siècle, puis aux parcs à la périphérie des villes dans les années 1980 et enfin, à la gestion différenciée des espaces verts ( $c f$. tableau 2).

Pour les OPP, ces regards ont également un impact. Les points de vue initiaux sont choisis selon les problématiques du territoire lors du comité de pilotage à partir d'un ensemble de clichés. Pour analyser les dynamiques urbaines, une sélection de ces points de vue est faite. Une même photographie peut servir plusieurs démonstrations. Ainsi, selon le récepteur, l'interprétation varie suivant ses propres besoins donnant à l'image différents schèmes idéologiques et culturels possibles.

Ce premier corpus choisi est complété par des photographies isolées effectuées spécifiquement pour le projet afin de montrer des préoccupations paysagères actuelles non identifiées dans le corpus disponible. Elles sont signifiées en bleu dans le tableau 1 Se pose la question de l'obsolescence des OPP comme du renouvellement régulier des points de vue initiaux pour aborder des thématiques nouvelles. Les porteurs d'OPP, s'imposant un protocole rigoureux, rephotographient des points de vue n'étant plus signifiants au regard de leurs problématiques. Dans un même temps, de nouvelles préoccupations émergent et ne sont pas traitées. Une évaluation doit être conduite régulièrement afin de maintenir un nombre raisonnable de reconductions tout en s'octroyant une certaine flexibilité dans le renouvellement des points de vue. Ce nombre est capital pour explorer le contenu photographique et l'intégrer au sein d'une analyse territoriale plus étendue.

27 Nonobstant ces hiatus, le corpus, par son hétérogénéité, permet d'entrecroiser diverses échelles spatiotemporelles facilitant la reconstitution des dynamiques paysagères. 
Tableau 1. Sujets d'intérêts selon les thématiques à travers le temps

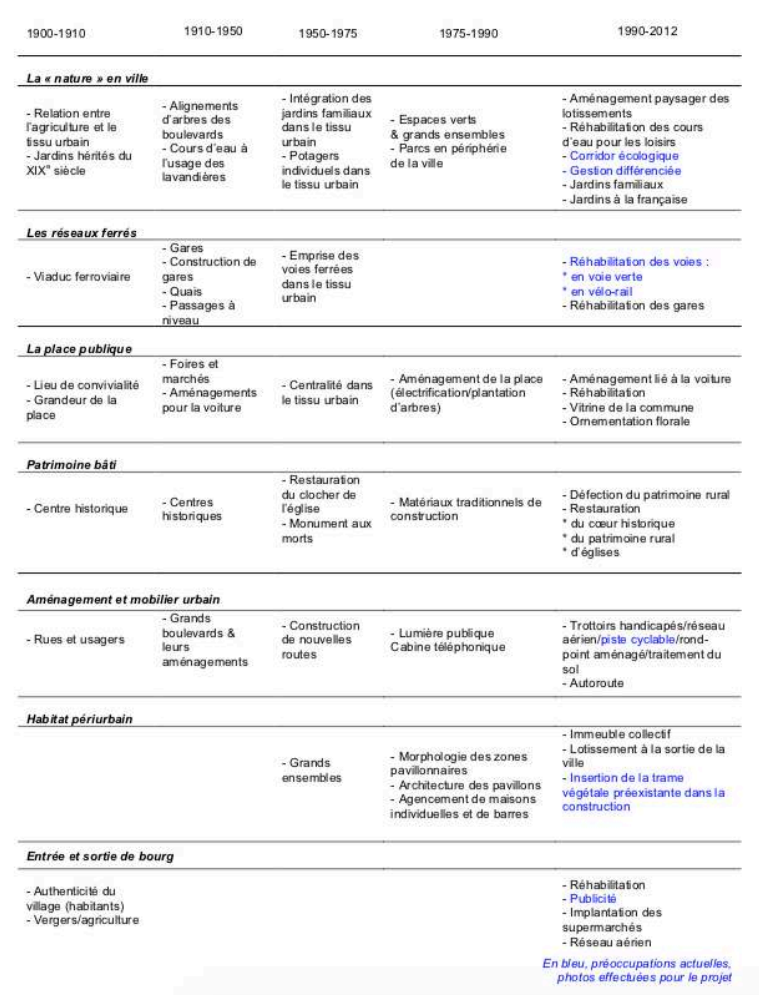

Ce classement, à partir du corpus étudié, explicite les sujets photographiés selon les époques.

\section{Analyse des dynamiques paysagères urbaines à partir du corpus photographique}

"Les dynamiques paysagères inscrivent les paysages dans des "trajectoires" avec leurs histoires passées et leurs transformations futures » (Davodeau, Toublanc, 2010, p. 4). Le corpus photographique illustre ces «trajectoires » et facilite l'analyse des dynamiques urbaines pour déterminer des scénarios séquentiels repris dans le bloc-diagramme évolutif.

\section{Des dynamiques paysagères générales aux dynamiques paysagères locales}

À partir de recherches bibliographiques, des dynamiques urbaines générales sont identifiées. Le corpus photographique est ensuite classé suivant sa promiscuité selon les dynamiques préétablies en amont. Ce basculement spatial des dynamiques générales aux dynamiques locales permet d'illustrer finement les processus évolutifs. Le tableau 2 indique l'ensemble des transformations visibles dans le corpus et représentatives des dynamiques régionales. Il renseigne également les changements d'échelles temporelles (avec un fond grisé, les couples diachroniques et avec un fond blanc, les OPP) et les typologies de points de vue. 
Tableau 2. Dynamiques visibles sur le corpus et représentatives des dynamiques régionales

\begin{tabular}{|c|c|}
\hline Points de vue médians & Points de vue proches \\
\hline \multicolumn{2}{|l|}{ Les dynamiques liées à l'habitat } \\
\hline Densification urbaine & - Densification urbaine \\
\hline $\begin{array}{l}\text { - Élalement urbain } \\
\text { - Mitage }\end{array}$ & - Etalement urbain \\
\hline $\begin{array}{l}\text { - Mitage } \\
\text { - Déliquescence du patrimoine rural }\end{array}$ & $\begin{array}{l}\text { - Conservation du patrimoine rural avec des } \\
\text { périodes de restauration }\end{array}$ \\
\hline - Disparition des vergers et des potagers à & - Réhabilitation du coeur historique \\
\hline larrière des maisons & - Réhabilitation des bâtiments publics \\
\hline $\begin{array}{l}\text { - Mitage } \\
\text { - Densification urbaine }\end{array}$ & - Densification urbaine \\
\hline \multicolumn{2}{|l|}{ Les dynamiques paysagères liées à la mobilit } \\
\hline $\begin{array}{l}\text { - Suppression du tramway/viaduc } \\
\text { - Diminution des espaces piétons } \\
\text { - Aménagement de la voirie (rue goudronnée, } \\
\text { parking, rond-point) }\end{array}$ & $\begin{array}{l}\text { - Aménagement de parking sur la place publique } \\
\text { et le long des voies } \\
\text { - Création de trottoirs } \\
\text { - Multiplication de la signalétique, des Abribus, } \\
\text { des poteaux de sécurité } \\
\text { - Aménagement des passages à niveau } \\
\text { - Réorganisation de la place publique } \\
\text { (délimitation des espaces selon les usagers) }\end{array}$ \\
\hline $\begin{array}{l}\text { - Aménagement de parking (covoiturage) } \\
\text { - Mise aux normes d'une } 2 \times 2 \text { voie }\end{array}$ & $\begin{array}{l}\text { - Traitement de la voirie (texture du } \\
\text { sol/marquage)/rond-point }\end{array}$ \\
\hline \multicolumn{2}{|c|}{ Les dynamiques paysagères liées aux fonctions économiques } \\
\hline & - Modifications des commerces dans les bourgs \\
\hline $\begin{array}{l}\text { - Agrandissement des usines agroalimentaires } \\
\text { - Modification des zones artisanales } \\
\text { (renouvellement des bâtiments, changement } \\
\text { d'enseignes, renouvellement de la publicité) }\end{array}$ & - Agrandissement des zones commerciales \\
\hline \multicolumn{2}{|l|}{ Les dynamiques paysagères liées aux espa: } \\
\hline $\begin{array}{l}\text { - Aménagement des cours d'eau en espace de } \\
\text { loisis } \\
\text { - Ajout de massifs fleuris, bacs fleuris }\end{array}$ & $\begin{array}{l}\text { - Electrification de l'espace public } \\
\text { - Multiplication du mobilier urbain } \\
\text { - Plantation d'arbres sur la place publique } \\
\text { - Apport de massifs fleuris, bacs fleuris } \\
\text { - Suppression des arbres des boulevards }\end{array}$ \\
\hline & $\begin{array}{l}\text { - Enfouissement du réseau aérien/transformateur } \\
\text { - Apport de massifs fleuris, bacs fleuris }\end{array}$ \\
\hline \multicolumn{2}{|c|}{$\begin{array}{l}\text { Les dynamiques paysagères liées aux espaces de transition (lisière entre espace privé et } \\
\text { espace public/entre espace rural et espace urbain) }\end{array}$} \\
\hline $\begin{array}{l}\text { - Aménagement des entrées et sorties de ville } \\
\text { (trottoirs, voirie) }\end{array}$ & $\begin{array}{l}\text { - Suppression des vergers et densification } \\
\text { urbaine dans les dents creuses aux entrées et } \\
\text { sorties de ville } \\
\text {-Enfrichement }\end{array}$ \\
\hline $\begin{array}{l}\text { - Création de supermarchés aux entrées et } \\
\text { sorties de villes } \\
\text { - Rénabilitition des entrées et sorties de villes } \\
\text { (ajout d'alitinements d'arbres, trottoirs, ronds- } \\
\text { points, enfouissement du réseau électrique) }\end{array}$ & $\begin{array}{l}\text { - Intégration de l'espace privé dans l'espace } \\
\text { public } \\
\text { - Enfrichement }\end{array}$ \\
\hline
\end{tabular}

Les dynamiques issues des couples diachroniques sont sur fond gris et les dynamiques issues des OPP sont sur fond blanc.

Par exemple, la densification urbaine, dynamique bien identifiée au-delà de la région Bretagne, est visible à travers le temps long et le temps court. Le couple diachronique de Saint-Brieuc vu de Plérin (cf. figure 6) est une traduction de ce processus, donnant à voir l'ampleur des changements en un siècle. 
Figure 6. Par classement analogique, les photographies peuvent illustrer la même dynamique en accentuant des spécificités locales

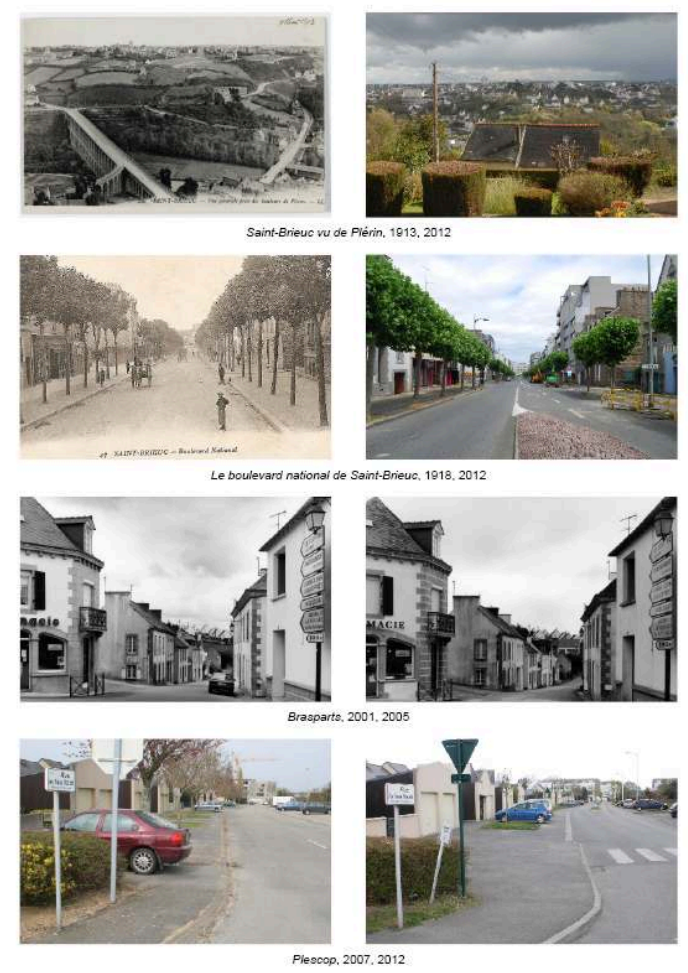

Sources (de gauche à droite) : Archives départementales des Côtes d'Armor, fonds 6 Fl, droits réservés/Emmanuel Poirier, Syndicat mixte du Pays de Saint-Brieuc, droits réservés/Association Ameno, droits réservés/Laboratoire Eso-Rennes/Jean-Christophe Ballot, Observatoire photographique national du paysage, ministère, Itinéraire $n^{\circ} 19$, PNRA, droits réservés/Jean-Christophe Ballot, Observatoire photographique national du paysage, ministère, Itinéraire $n^{\circ} 19$, PNRA, droits réservés/ David Ledan, Observatoire photographique du paysage, Siagm-projet du PNR golfe du Morbihan, droits réservés/David Ledan, Observatoire photographique du paysage, Siagm-projet du PNR golfe du Morbihan, droits réservés.

31 Avec un autre couple diachronique de point de vue proche du boulevard national de Saint-Brieuc (cf. figure 6), la densification urbaine se caractérise par la destruction de maisons traditionnelles au profit de petits collectifs. Ces formes urbanistiques évoluent à travers différentes temporalités à la fois de manière diachronique avec un réseau viaire conservé et à la fois de manière synchronique où la « rupture de la forme dans le temps se constate lorsque l'intervention sociale sur le milieu crée un état nouveau " (Chouquer, 2000, p. 125). La transformation s'effectue simultanément avec l'encouragement des politiques publiques à densifier la ville en autorisant la construction d'immeubles de 21 mètres dans les années 1970. Les séries OPP ont l'avantage de déterminer finement l'échelle temporelle des mutations comme à Brasparts ( $c f$. figure 6) où une maison individuelle s'insère dans le paysage urbain entre 2001 et 2005. À Plescop, la densification est signifiée par l'implantation d'immeubles collectifs ( $c f$. figure 6). Ces quatre séries qualifient une même dynamique apportant pour chacune d'entre elles des informations singulières.

Certains espaces comme les places publiques traduisent un ensemble de dynamiques (liées à l'habitat, à la mobilité, aux fonctions économiques, aux espaces publics). Ainsi, les points de vue initiaux issus des couples diachroniques mettent en lumière la disproportion des places publiques qui sont dépourvues de tout aménagement au début 
$\mathrm{du} \mathrm{xx}^{\mathrm{e}}$ siècle. Cette organisation est due aux fonctions économiques de la place qui accueillait les foires et les marchés. Cent ans après, les places sont réorganisées pour rationnaliser l'espace. De nombreux éléments apparaissent: ronds-points, réseau aérien, places de parking, trottoirs, signalisation, etc. Les commerces, présents autour de la place publique, sont renouvelés, voire en augmentation alors que selon les dynamiques générales, l'arrivée des supermarchés affecte le nombre de commerces de proximité. L'analyse du corpus photographique peut exposer des faits contradictoires. Les séries OPP privilégient les préoccupations actuelles liées à la place publique par la valorisation des opérations de réhabilitation (enfouissement du réseau aérien, diminution des places de parking au profit d'ornementation végétale). Ces transformations se caractérisent par une diversité de «faire » dépendant des politiques locales. Les couples diachroniques révèlent des dynamiques très frappantes en Bretagne avec la suppression des vergers, la densification urbaine, l'aménagement de la voirie. Les séries OPP exposent les requalifications des espaces transitoires et l'implantation de supermarchés. Concernant la prégnance de la publicité des entrées et sorties de ville, phénomène généralisé en Bretagne, il est paradoxalement très peu présent dans les séries des OPP étudiés.

À partir d'une dynamique ou à partir d'une typologie spatiale (entrée de ville, place publique), les similitudes du corpus photographique font émerger une représentativité à l'échelle régionale en maintenant les singularités locales. L'étude visuelle apporte des informations fines sur l'insertion des éléments de paysage au sein des dynamiques identifiées, facilitant la compréhension de l'évolution d'un paysage urbain type. La régularité des séries OPP précise l'échelle temporelle des aménagements affectant le paysage bien que certaines problématiques ne soient pas abordées. Les OPP avec un point de vue proche veillent à l'état d'avancement des projets d'aménagement. Les points de vue médians permettent d'aborder plus facilement les interrelations. Cette méthode d'analyse n'est pas appropriée pour répondre à toutes les finalités allouées aux OPP notamment dans l'identification « des rôles des différents acteurs » dans ces mutations (Meeddm, 2009, p. 6).

\section{De l'élément de paysage aux usages}

34 L'évolution de certains éléments de paysage, particulièrement à travers les couples diachroniques, révèle différents usages non ostensibles à travers les recherches bibliographiques. Pour exemple, les alignements d'arbres le long des boulevards plantés à même le trottoir au début du $x^{e}$ siècle ont un rôle d'ombrage pour les piétons. Leur suppression (due notamment à la graphiose) permet de réduire la largeur des trottoirs pour aménager des parkings. Aujourd'hui les alignements d'arbres replantés ont une fonction d'ornementation et de purification de l'air.

De même, l'implantation du mobilier dans le tissu urbain traduit des pratiques liées à un contexte historique. La place de la République à Rennes dans les années 1930 est parée de bancs publics qui font face aux jardins d'agrément. Une dizaine d'années après, la place est réaménagée pour s'adapter aux besoins liés à la voiture et les bancs sont désormais orientés vers la voie de circulation.

Le corpus donne à voir également des aménagements non adaptés aux usages comme les stationnements anarchiques sur les voies publiques pointant alors des espaces 
inadéquats aux besoins de parking. La photographie, indicateur de formes mais également d'usages, renouvelle des connaissances sur les pratiques liées aux paysages.

\section{Articulation possible avec les représentations sociales des acteurs du territoire?}

Les études à partir des séries photographiques n'ont pas seulement comme objectif de décrire et d'appréhender les dynamiques paysagères de manière factuelle, elles visent également à comprendre les représentations sociales des dynamiques paysagères auprès des acteurs du territoire (élus, professionnels, habitants). Certaines recherches constatent que le recours aux séries rétrospectives durant les enquêtes sociales stimule peu les discours des populations sur les dynamiques paysagères comme l'exposent les premiers résultats sur la fabrique des Observatoires Homme-Milieu des Pyrénées-Haut Vicdessos (Davasse et al., 2012). Dans cette même perspective, Dominique Henry explicite clairement que les photographies répétées "n'avaient qu'un rôle de second plan» lors de ses entretiens où «la chronique photographique des évolutions paysagères ne suscitait pas d'engouement particulier » (Henry, 2012, p. 122). Les séries rétrospectives avec des intervalles de temps éloignés sont-elles difficilement appréhendables comparées aux séries prospectives avec des intervalles de temps courts ? La lecture de ces séries nécessite-t-elle un apprentissage ?

Il reste donc à évaluer précisément le rôle des OPP dans l'analyse des représentations sociales des dynamiques paysagères en comparant des entretiens effectués à partir de séries photographiques et des entretiens sans support photographique; travaux en cours au laboratoire Eso-Rennes ${ }^{15}$.

\section{Conclusion}

La rigidité méthodologique supposée des OPP entraîne un investissement considérable dans la conception de l'outil au détriment de l'analyse accordant « une autonomie » aux images mais «ne faisant pas pour autant sens " (Briffaud, 2000, p. 65). À travers cette contribution, il est constaté que les méthodes d'analyse des dynamiques paysagères à travers les OPP sont diverses et complémentaires. L'enjeu de ces analyses tient dans la délimitation de l'objet de recherche. Sous cette masse documentaire, des choix sont à effectuer pour extraire le corpus approprié et pour adapter une ou des méthodes en assumant le risque de perdre des informations. Au regard de la multiplicité des dynamiques paysagères, il semble difficile d'établir une méthode commune satisfaisant le plus grand nombre et sur le long terme. Avec des perspectives de facilitation des méthodes possibles (identification des éléments et de leurs changements, intentionnalité du photographe, géoréférencement des séries, etc.), la construction d'un fonds mutualisé des photographies du paysage encouragerait leur exploitation.

Nous avons montré que le corpus photographique sur le paysage urbain est représentatif des dynamiques urbaines de Bretagne en exprimant des spécificités locales et des usages parfois inattendus. Le degré de précision des photographies vues du sol apporte des connaissances autres que celles issues des cartes, des données statistiques et de télédétection. 
41 L'étude des séries OPP soulève des questionnements sur leurs attributions opérationnelles. Les séries sur les paysages urbains sans changement significatif contribuent à la mémoire des paysages, cependant les reconductions quinquennales voire décennales sont peut-être suffisantes afin de réduire les coûts. Certaines séries assurent une veille sur des projets d'aménagement. Cependant après l'achèvement de l'aménagement, après la constatation de ces effets sur les usages, ne faut-il pas archiver cette série et réorienter le point de vue vers de nouvelles préoccupations paysagères ?

De plus, la prise de vue et le cadrage conditionnent la lecture. Les cadrages « objectivants » et les cadrages mixtes semblent plus aptes à renseigner sur le paysage factuel. Les points de vue proches pourraient être contextualisés par des points de vue médians pour mieux appréhender les dynamiques paysagères et pour mieux « explorer toute l'épaisseur des paysages observés » (Carré et al., 2011, p. 3).

43 En dépit de ces questionnements, les OPP, par leur valorisation, sont des outils didactiques et mémoriaux efficaces pour sensibiliser les élus et les populations aux problématiques paysagères vers des réflexions communes sur la construction du paysage de demain.

\section{BIBLIOGRAPHIE}

Briffaud, S., « Enjeux et problématiques de la représentation du changement paysager », dans « Itinéraires croisés, rencontres de l'observatoire photographique du paysage du 24-35 septembre 1999 à Rochefort », ministère de l'Aménagement du territoire et de l'Environnement, 2000, p. 59-68.

Carré, J., Davasse, B., Métailié, J-P., « Vers un observatoire partagé des paysages du Parc national des Pyrénées, méthodes et matériaux pour analyser et illustrer l'évolution des paysages sur le territoire du Parc ", issu des Journées scientifiques du parc national des Pyrénées du 7 et 8 octobre 2011, Villelongue, 2011, 4 p.

Carré, J., Métailié, J-P., « De los paisajes de ayer a los paisajes de mañana. Metodología de un observatorio fotográfico para el análisis de las dinámicas paisajísticas : el valle de Vicdessos, Pirineos de Ariége (Francia) », Cuadernos geográficos, nº 43, 2008, p. 123-149.

Chouquer, G., L'Étude des paysages. Essais sur leurs formes et leur histoire, Paris, Édition Errance, 2000, $208 \mathrm{p}$.

Conseil de l'Europe, Convention européenne du paysage, Florence, 2000.

Coutanceau, A., «Étude de la complémentarité entre les dynamiques paysagères observées dans les séries photographiques de l'OPNP et les documents de la connaissance et de la planification », mémoire de fin d'étude en ingénierie des territoires, Angers, Agrocampus Ouest, 2011, 114 p.

Davasse, B., Briffaud, S., Carré, J., Henry, D., Rodriguez, J-F., « L'observatoire environnemental au prisme du paysage. Dynamiques paysagères, actions territoriales et représentations sociospatiales contemporaines dans le territoire de l'OHM Pyrénées-Haut Vicdessos ", Sud Ouest européen, $\mathrm{n}^{\circ} 33,2012$, p. 57-68. 
Davodeau, D., Toublanc, M., « Le paysage-outil, les outils du paysage, principes et méthodes de la médiation paysagère ", issu des conférences sur les outils pour décider ensemble, aide à la décision et gouvernance, 25 au 25 octobre 2010, Montpellier, 2010, 17 p., URL : URL : http:// hal.archives-ouvertes.fr/docs/00/78/81/55/PDF/Outils_paysage.pdf.

Dérioz, P., Béringuier, P., Laques, A-E, « Mobiliser le paysage pour observer les territoires : quelles démarches, pour quelle participation des acteurs? ", Développement durable et territoires, vol. $1, \mathrm{n}^{\circ} 2$, mis en ligne le 25 novembre 2010, URL : http://developpementdurable.revues.org/ 8682.

Griselin M., Ormaux, S., « Pour une approche paysagère des espaces polaires ", Norois, 2005, mis en ligne le 13 août 2008, URL : http://norois.revues.org/682.

Grison, L., « Le vieillissement des lieux, photographier, décrire-écrire », L'Espace géographique, $\mathrm{n}^{\circ}$ 3, 1998, p. 276-279.

Henry, D., « "Entre-tenir la montagne” : paysage et ethnogéographie du travail des éleveurs en montagne pyrénéenne : hautes vallées du Gave de Pau, de Campan et d'Oueil-Larboust », Toulouse 2, thèse de doctorat en géographie et aménagement, 2012, $416 \mathrm{p}$

Le Dû-Blayo, L., Le Paysage en Bretagne. Enjeux et défis, Quimper, Éditions Palantines, 2007, 350 p.

Lobet, J., Nederlandt, N., Rosillon, F., « Mise en place d'un observatoire du paysage dans le bassin transfrontalier Semois-Semoy ", rapport final du contrat de rivière Semoy-Semois, 2006, 67 p.

Luginbühl, Y. (dir.), Analyse des transformations des paysages présentés dans les clichés de l'Observatoire photographique du paysage. Utilisation du logiciel capteur de plan, Paris, UMR Ladyss CNRS/UMR Prodig CNRS, 1999, 23 p.

Meeddat, Itinéraires photographiques. Méthode de l'observatoire photographique du paysage, Paris, $2008,74 \mathrm{p}$.

Meeddm, L'Observatoire photographique au service des politiques du paysage, actes du colloque européen du 13 et 14 novembre 2008, Paris, 2009, 196 p.

Mendibil, D., « Dispositif, format, posture : une méthode d'analyse de l'iconographie géographique », Cybergeo, mis en ligne le 12 mars 2008, URL : http://cybergeo.revues.org/16823.

Métailié, J.-P., « Le photo géographe et l'histoire des paysages », dans Séquences paysages. Revue de l'Observatoire photographique du paysage, Paris, Éditions Hazan, 1997, p. 91-95.

Michelin, Y., Le Jardin de vulcain : paysage d'hier, d'aujourd'hui et de demain dans la chaîne des Puys du Massif central français, Paris, Éditions de la Maison des sciences de l'homme, 1995, 155 p.

Sevenant, M., Antrop, M., « Landscape representation validity: a comparaison between on-site observations and photographs with different angles of view », Landscape Research, vol. $36, \mathrm{n}^{\circ} 3$, june 2011, p. 363-285.

Sgard, A., « Le paysage dans l'action publique : du patrimoine au bien commun », Développement durable et territoires. Économie, géographie, politique, droit, sociologie, vol. 1, n² 2, 2010, URL : http:// developpementdurable.revues.org/8565.

\section{NOTES}

1. Ministère de l'Écologie, de l'Énergie, du Développement durable et de la Mer, aujourd'hui, ministère de l'Écologie, du Développement durable et de l'Énergie, se référer au site Internet: www.side.developpement-durable.gouv.fr/. 
2. Conseil d'architecture, d'urbanisme et de l'environnement.

3. Syndicat intercommunal d'aménagement du golfe du Morbihan.

4. Ce projet, dirigé par Laurence Le Dû-Blayo porte sur l'évolution des dynamiques urbaines (Caroline Guittet), de vallées et du littoral balnéaire (Caroline Le Calvez). Il a été réalisé par « des mondes singuliers » et est disponible sur le site Internet: http://evolution-paysage.bretagneenvironnement.org/.

5. Les éléments de paysage sont des « objets matériels qui ne peuvent pas être considérés comme des systèmes du point de vue paysager mais qui ont des caractéristiques paysagères, c'est-à-dire qu'ils sont perçus au travers de filtres culturels ». Roche, A., Les Unités et les Structures paysagères dans les atlas de paysages, Paris, ministère de l'Écologie et du Développement durable, 2007, p. 14.

6. Les structures paysagères «correspondent à des systèmes formés par des objets, éléments matériels du territoire considéré et les interrelations, matérielles ou immatérielles, qui les lient entre eux et/ou à leur perception par les populations. ». Seguin, J.-F., Des composantes du paysage : Unités, structures, éléments, Paris, Meeddatt, 2005, p. 3.

7. Unité mixte de recherche « Théoriser et modéliser pour aménager ».

8. Les appareils photographiques sont de haute qualité pour supporter le milieu physique, leurs positionnements font l'objet de calculs complexes afin de recouvrir toute la zone étudiée.

9. Travail réalisé dans le cadre de l'observatoire photographique de l'autoroute 89 , se référer au site Internet : poptmc.free.fr.

10. Ibid.

11. Dervieux, A., "Que peuvent-nous dire les anciennes photographies sur les changements paysagers? ", dans le cadre des journées d'étude "Comment les images de paysage interrogent les territoires ?», 9 et 10 mars 2004, École nationale de formation agronomique de Toulouse (Enfa).

12. Le parc naturel transfrontalier du Hainaut, le parc naturel régional Scarpe-Escaut et le parc naturel des Plaines de l'Escaut se sont associés pour mettre en place une méthodologie commune, les éléments présentés ici proviennent de documents de travail en cours.

13. Le cadrage est la délimitation du champ visuel de la photographie.

14. Le point de vue se définit spatialement, c'est l'endroit à partir duquel le photographe enregistre la photographie.

15. Notamment dans le cadre de la présente thèse sur l'analyse des dynamiques paysagères à partir des OPP en Bretagne et dans le cadre du stage en cours de Simon Monthulé, tous deux dirigés par Laurence Le Dû-Blayo au laboratoire Espaces et Sociétés.

\section{RÉSUMÉS}

Les séries photographiques diachroniques sont des ressources didactiques pour appréhender les dynamiques paysagères. Plusieurs expériences d'horizons disciplinaires variés l'ont attesté par l'emploi de méthodes quantitatives et/ou qualitatives rigoureusement menées. L'exemple de documents sur les dynamiques urbaines en Bretagne permet ici d'étudier les potentialités du corpus photographique et des méthodes d'analyse dans la connaissance des mutations paysagères. L'exploitation des Observatoires photographiques du paysage (OPP) et d'un fonds iconographique complémentaire, au moyen d'une approche qualitative, traduit visuellement de manière fine les dynamiques paysagères. Ce corpus est représentatif des dynamiques régionales 
de l'échelle séculaire à l'échelle pluriannuelle en soulevant des spécificités locales et des pratiques en lien avec le paysage.

Diachronous photographic series are didactic resources for understanding landscape dynamics. In fact, several experiments, using quantitative and qualitative methods, attest to this interest in analysing photographs. The example of documents on urban dynamics in Brittany (France) makes it possible to study potentials of both corpus and methods. The utilization of photographic observatories of landscape (Observatoires photographiques du paysage, OPP) and of an iconographic complementary fund with a qualitative approach gives a detailed reflection of landscape dynamics. In other words, this corpus is representative of the regional dynamics from the century scale to the multiannual scale through identification of local specificities and practices in connection with the landscape.

\section{INDEX}

Mots-clés : dynamique paysagère, Observatoire photographique du paysage, perception du paysage, sensibilisation au paysage, Bretagne

Keywords : landscape dynamics, photographic observatories of landscape, landscape perception, landscape awareness, Brittany (France)

\section{AUTEURS}

\section{CAROLINE GUITTET}

Caroline Guittet est doctorante en géographie, laboratoire ESO-Rennes, UMR CNRS Eso Rennes 6590, université Rennes II.

caroline.guittet[at]uhb[dot]fr

\section{LAURENCE LE DÛ-BLAYO}

Laurence Le Du est maître de conférences en géographie, laboratoire Eso-Rennes, UMR CNRS ESO Rennes 6590, université Rennes II.

laurence.ledu[at]uhb[dot]fr 